DR THOMAS VOLZ

Associate Professor, Department of Physics and Astronomy, Macquarie University, Australia

..........

\section{FIELD OF RESEARCH}

Quantum Optics, Quantum Engineering, Quantum Materials

that the right materials are used to perform specific functions. Nanodiamonds are an example. Somewhat ironically, to gain a greater understanding of nanodiamonds' or other solid materials' usefulness in a given application, researchers need to use quantum technologies to study them. Understanding the behaviour of quantum emitters in solids can provide insight into the material and its fundamental behaviour. "It is worth noting that we are in the midst of what we call the Second Quantum Revolution and still need to learn a lot about the different quantum materials out there," says Thomas. "Studying quantum emitters in solid materials can provide us with new material platforms for implementing a range of new quantum technologies."

\section{WHAT ARE THE POTENTIAL} APPLICATIONS OF QUANTUM LIGHT? In its most extreme form, quantum light consists of single identical photons that arrive in time, one after the other, like the pearls on a string. Not all light is like this - sunlight, for instance, consists of photons that tend to come in pairs, while laser light exists somewhere between the two extremes. Laser photons come at completely random times and have no correlation with each other. Even so, lasers are one of the most powerful technologies in the world today.

To generate quantum light, a quantum emitter is needed, which is where Thomas's studies come in. Quantum light is a key resource for a variety of potential quantum information applications, such as building a quantum internet and performing quantum computations with light.

\section{WHAT OTHER ACTIVITIES IS THOMAS \\ ENGAGED IN?}

In addition to his work on quantum emitter systems, Thomas has co-founded a start-up company called Redback Systems. The team

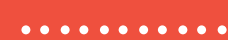

\section{RESEARCH PROJECT}

Thomas is engaged in a variety of quantum research projects, typically involving quantum emitters and light. His studies will help understand various materials, their fundamental behaviours and suitability for a range of technological applications that will change the world.

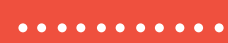

\section{FUNDER}

Australian Research Council Centre of Excellence for Engineered Quantum Systems (EQUS)

at Redback Systems is building advanced optical spectrometers that have their origin in astronomy (two of Thomas's co-founders are astronomers with experience in building some of the best and most expensive spectrometers in the world). "With many visitors coming through our lab and asking where one can buy these spectrometers, we realised there was a commercial opportunity and decided to go for it," says Thomas. "We were greatly supported by the ARC Centre of Excellence for Engineered Quantum Systems (EQUS) and Australia's national science organisation CSIRO through its $\mathrm{ON}$ programme. This enabled us to form a viable company and we shipped our first product in January 2021."

If all that was not enough, Thomas is also the Principal Investigator of QMAPP, more details of which can be found in the following pages. It is fair to say that regardless of how small the focus of quantum science is, Thomas is involved in a very large number of different projects!

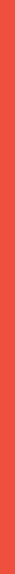

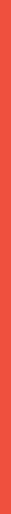

as using predictions that are a probability of
possible outcomes rather than something more definitive.

Still, despite or rather because of the bizarre quantum science boasts huge potential for changing the world in a range of ways. For heart of a quantum computer. It is with this in mind that Dr Thomas Volz, based at Macquarie quantum experiments involving quantum WHAT ARE QUANTUM

Quantum emitters emit single photons (the basic unit of all light) one by one. The atom with two distinct energy levels. When absorb a photon, go to an excited state and "As well as isolated atoms in free space, solid-state materials can be host to so-called typically consist of either electron-hole pairs in . solid crystal, as is the case for colour centres in diamond." [See Lachlan Rogers' article: https:/ quantum-scientists-best-friend].

BEHAVIOUR OF QUANTUM EMITTERS IN MATERIALS?

If our future world is going to make use of 


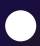 ABOUT QMAPP}

Thomas is Principal Investigator of the Quantum Materials and Applications Group (QMAPP), a group focused on research activities in quantum physics, nanotechnology and materials science. QMAPP is part of the Physics and Astronomy Department at Macquarie University and is composed of a small team of seasoned researchers, as well as early career researchers and students who are just starting out on their pathway.

\section{WHAT FACILITIES DOES QMAPP}

\section{HAVE ACCESS TO?}

Given the nature of its scientific enquiries, it is vital that the team has access to stateof-the-art facilities. "We have a number of high-power, widely tunable, narrow-line width lasers for manipulating the quantum systems we are dealing with, as well as stateof-the-art single-photon detectors that are able to detect single photons with very high efficiency and with a few tens of picoseconds $(1 / 1,000,000,000,000$ seconds) time resolution," explains Thomas. "We also have special, optically accessible fridges for carrying out experiments at helium temperatures, which are four degrees above absolute zero."

HAVE THE TEAM AT QMAPP HAD ANY MAJOR ACHIEVEMENTS?

Yes! In 2019, they were able to report on a key scientific breakthrough, which was centred on the 'emergence of quantum correlations from interacting fibre cavity polaritons' and was published in Nature Materials. "The observation we reported on was predicted in 2006 and was (and still is) one of the key goals in the field of exciton polaritons. Essentially, we report on a way to create quantum light from semiconductor electron-hole pairs that does not require the presence of single quantum emitters in the material," says Thomas. "I was personally thrilled by the outcome since this was a project I started working on when I was a postdoctoral researcher at ETH Zurich in Switzerland, back in 2009!"

The findings took ten years from inception to publication, but Thomas considers it to be one of the key achievements of his career so far. This highlights the hard work that goes into a scientific career and how perseverance leads to great things. That said, scientific discoveries are not the only significant achievement. Thomas alludes to the fact that the group dynamics at QMAPP are really good - collaboration and working alongside like-minded scientists is a perk of the job that you too could enjoy one day.

\section{HOW TO BECOME A QUANTUM SCIENTIST}

- STEM Learning has a wealth of resources for those interested in quantum technology. Although many of the papers, on topics such as diffraction, cryptography and laser cooling, are designed for teachers to use, they can be accessed by anybody: https://www.stem.org.uk

- Forbes has put together six things we should all know about quantum physics. It is a fascinating read and should enable you to read around specific areas within the field: https://tinyurl.com/2sryvvu4

- There are so many different roles within quantum science that an estimated salary is difficult to provide. However, for a research scientist in quantum information science, the average salary is the equivalent of around AUS $\$ 100,000$. It is worth noting that quantum physics is such a niche field, and one that will revolutionise the world before long, meaning that people with expertise and knowledge in this field will be highly sought after.

\section{THOMAS'S TOP TIPS}

OI There are a few key traits that are important for a career in science, such as curiosity and an ability to think logically. If you can develop those skills as soon as possible, it will stand you in good stead throughout your career.

02 Quantum science requires very good maths skills, particularly as physics is a quantitative science. Do everything you can to hone your understanding of maths and pay attention in class!

03 The ability to present in front of others and defend key arguments when questioned are important to succeed in science. In addition, excellent writing skills will enable successful grant and publication writing.

\section{PATHWAY FROM SCHOOL TO QUANTUM SCIENCE}

Thomas recommends students think about developing a broader range of skills other than simply focusing on maths and physics (which are essential to quantum science). It might be worth considering studying IT/programming and English, which will be useful for writing up your findings for publication.

You will need a degree in a relevant subject for postgraduate study such as mathematics and/or physics.

https://study.com/articles/quantum_physics_degree_programs_requirements.html 


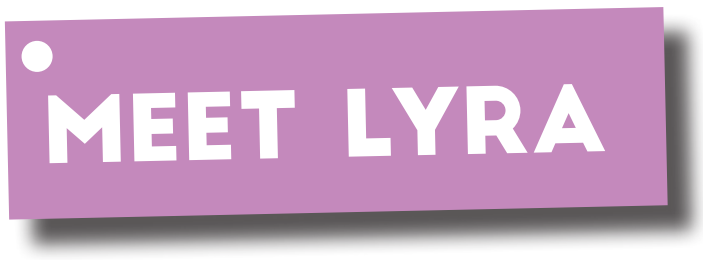

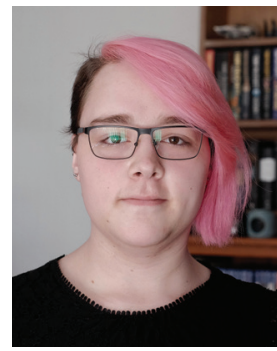

Lyra Cronin has just completed her Master of Research (MRes) degree, which means she was able to undertake her studies in quantum optics as part of the QMAPP team.

I loved English and physics during high school. This was mainly because of the wonderful teachers I had. In my first year at university, I did both linguistics and physics subjects, and because of the experience studying physics, it made me more certain that this is the path I wanted to follow.

\begin{abstract}
A large part of my motivation for working in this field is to make the world a better place. If by working steadily at research I can help further our understanding of the universe we live in, then there is no other way I would prefer to spend my time.
\end{abstract}

My current work is focused on the development and exploration of a maser system. This is a laser that operates at microwave frequencies and is realised by using diamond. While masers have been around for decades, and are used commonly in deep space communication, GPS satellites and high accuracy timekeeping, the existing systems are inaccessible for most other fields due to their cost and operating requirements.

I finished my MRes programme at the end of 2020. I started the maser project during my MRes thesis and will continue working on the same project over the next few years as part of my PhD. This has been my goal for some time, and I am still not sure where I will go afterwards - but, whatever I do, I will want to continue doing research.

I would certainly encourage others to study physics and quantum optics. Little in my life has been as rewarding as conducting research and even though it can be difficult at times, I have never doubted that this is the right path for me. Even outside of academia and research, a background in quantum physics means you are incredibly well positioned to be at the forefront of a new industry.
I would also encourage women and people from underrepresented backgrounds to consider studying physics. In the past, physics has not been especially welcoming for many people, but this is changing rapidly thanks to the efforts of many within the field. Macquarie University, in particular, has been wonderful, and has come so far so quickly. There is an idea that quantum physics is particularly difficult, but it is not inherently more difficult than any other field of science. Anybody can pursue a career in quantum science if they want to.

If I could speak to my younger self, I would say that getting a $\mathrm{PhD}$ is possible. Throughout the early years of university, I struggled with severe depression - I had to stop studying for a year and try to recover. Even then I had to study part time for most of my undergraduate degree. During that time, I also came out as trans and began transitioning, which was not easy. Ultimately, even with these struggles, I have been able to get to where I wanted, and it has been one of the best periods of my life. 